\title{
Understanding gel electrophoresis
}

Pulsed-field electrophoresis, the newest version of a standard technique, has become widely used for the sieving of large DNA molecules long in advance of an understanding of exactly how it yields useful results.

ELECTROPHORESIS may be one of the most widely used techniques of molecular biology, but its practice is still a craft. Some researchers, or their technicians, have acquired reputations for particular skill in the preparation of gels, or in the interpretation of the records won this way. And while the principles of gel electrophoresis are simple enough - the rate at which protein or DNA molecules are dragged through the aqueous interstitial network running through an inert polymer skeleton by an electric field depends on the length of the molecules, often a good proxy for mass - the limitations of the technique have usually been discovered the hard way (by experiment) before they have been accounted for by calculation.

That is why major innovations in gel electrophoresis have usually also arisen empirically. That is notably the case with the latest development, that of pulsedfield electrophoresis, in which the direction of the forcing electric field alternates at regular intervals between a forward direction and one that may be diametrically opposite or even include a substantial orthogonal component. (The technique, due to David C. Schwartz and Charles R. Cantor, is described in Cell 37, 67; 1984.) The technique has made it possible to separate larger molecules than are accessible when the electric field has a single direction - up to roughly 2 million nucleotide bases, compared with the limit of about 30,000 bases for the conventional technique. But why?

Hand-waving explanations are plentiful, but throw little light on how to optimize the new technique for the separation of molecules of particular size and conformation. Because the applied electric field (usually no greater than a few volts per centimetre) is invariably in the plane of the thin gel, the problem is that of the migration of long molecules in two dimensions. Most of the medium is aqueous, with the polymer skeleton (polyacrylamide or agarose) represented as a random scattering of physical obstacles which long molecules cannot penetrate but on which they can become entangled. Because the medium is aqueous, DNA molecules therein are uniformly charged along their length. In an electric field, they may be expected to drift in that direction. But at what rate?

This is where the fun begins. Suppose that, against all the odds, a DNA molecule is already stretched in the direction of the electric field, which in the simplest case is constant. The molecule has a leading end (the 'head') and a trailing end (the 'tail'). The head must find a path through the solid obstacles randomly presented by the gel, and the remainder of the molecule must, snakelike, follow (whence the description of the movement as 'reptation').

The simplest view of what happens is to suppose that the molecule moves within a tube, more or less in the direction of the field - that defined by the path sought out by the head of the molecule. But there is an obvious difficulty. The unthinking head, subject to unavoidable thermal fluctuations, will hunt in one direction and another for a path through the obstacles in its way, but the path for the remainder of the molecule is already defined, so that the speed at which the tail end sections move behind the head will be determined only by the time taken for thermal fluctuations, as biased by the electric field, to carry them along the tube.

The mobility of the molecule as a whole will be determined by the likelihood that its successive parts will move in the direction of the electrical bias. This is essentially a random walking problem, so that the mobility is proportional to the square root of the length (or mass) of the molecule. So the resolution of electrophoresis should be inversely proportional to the length of the molecules whose separation is attempted.

Sadly, there are complications. For one, even on the assumption that the migration of the molecule represents motion along some notional tube, its trailing sections will travel faster than the head, so that a molecule that began as a stretched-out string will become a more tightly knit conglomerate. The longer the molecule or the stronger the electric field, the more quickly this will happen, when the mobility will be determined by the resistance to the passage of the tangle, whose dimensions will vary only slowly (say, as the one-third power) with length. The mobility of large molecules will be anomalously greater than expected, and sufficiently large molecules will be indistinguishable from each other by their mobility.

Simple (indeed, over-simple) as it is, this picture gives a hint of how pulsed-field electrophoresis may help with the resolution of larger molecules. If the field is reversed, the trailing tangle will be partly unravelled, restoring molecular mobility at least for a time. Obviously, the reversed field must not last for longer than the direct field, or there would be no resolution of even short molecules unaffected by the tangling of trailing molecular sections.

Unfortunately, this picture is a drastic simplification, which takes no account of how molecules become entangled with the gel lattice. So, for the past five years, there has been a steady sprinkling of attempts to model the movement of polymer chains through gel lattices, supposed to consist of regular arrays of obstacles. The general idea is to represent the molecule by a notional string of beads, each carrying an electric charge, which may be connected inelastically or (more cleverly) by elastic springs. The problem is to estimate the rate at which such a structure moves through the lattice of obstructions.

J. M. Deutsch and T. L. Madden were among the first to show that there may be a curious and unexpected oscillation of a normally migrating polymer chain between the extended and tangled structures (J. Chem. Phys. 90, 2476; 1989). Now, Monica Olvera de la Cruz, Dilip Gersappe and Edward O. Shaffer from Northwestern University, Illinois, have described an even more elaborate calculation (using a Cray machine) that throws a little more light on what happens in pulsed-field electrophoresis (Phys. Rev. Lett. 64, 2324; 1990).

The oscillation between the bunched and extended configurations shows up clearly in calculations of the instantaneous radius of gyration (root mean square radius) of the migrating molecules. The beneficial effects of intervening pulses of electric field in a transverse direction show up both in calculations of molecular mobility and in snapshots of the simulations: a transverse field opens up tangled structures, assisting their migration in the principal direction (which nicely confirms the pictures reported last year by Schwarz and Koval in Nature 338, 520; 1989). It is even possible qualitatively to relate the relative duration of the direct and transverse fields to the length of the large molecules for which the elecrophoretic resolution will be greatest. Yet there is a long way to go before the problem can be said to be neatly tidied up — by which time, no doubt, there will be other innovations to account for.

John Maddox 